\title{
GERENCIAMENTO DE RISCO NA COMERCIALIZAÇÃO DO ARROZ
}

\author{
Alan Figueiredo de Arêdes \\ Doutor em Economia Aplicada/UFV \\ Departamento de Ciências Econômicas/UFF/Campos dos Goytacazez. \\ aredess@yahoo.com.br
}

\section{RESUMO}

O gerenciamento de risco na comercialização do arroz é de grande importância na minimização das perdas financeiras e na melhor alocação dos recursos produtivos da atividade. Nessa perspectiva, o estudo faz uma análise do risco da atividade orizícola aplicando a métrica Value at Risk (VaR). Especificamente, o artigo teve dois objetivos. O primeiro foi avaliar o nível de risco que os produtores de arroz estão expostos no processo de comercialização da commodity. O segundo objetivo, por sua vez, consistiu em avaliar a aplicabilidade da métrica $\mathrm{VaR}$ na mensuração do risco de mercado do arroz identificando qual a melhor maneira de se estimar o risco da commodity, a saber: método paramétrico ou não paramétrico. Assim, a metodologia e a forma de análise dos resultados baseiam-se na mensuração do nível de risco de mercado do arroz usando a métrica $\mathrm{VaR}$, empregando os métodos de cálculo paramétrico e não paramétrico. De acordo com os resultados, conclui-se que os agricultores estão expostos a um alto risco de oscilação de preços, o que pode levar a perdas financeiras e a descapitalização do setor. Adicionalmente, conclui-se que a métrica usada é uma valiosa ferramenta na gestão de risco e que, para o caso do arroz, apenas o método não paramétrico é indicado para a estimação do VaR, uma vez que a série de preços não apresentou uma distribuição normal.

Palavras-chave: arroz, preço, valor em risco.

\begin{abstract}
Risk management in the marketing of rice is of great importance for minimizing financial losses and better allocation of production resources. From this perspective, this study analyzes the risks of rice production by applying the measure Value at Risk (VaR). Specifically, this article had two objectives. The first was to assess the level of risk that rice farmers are exposed to in the process of marketing the commodity; and the second was to assess the applicability of the $\mathrm{VaR}$ for measuring the market risk of rice, identifying how best to estimate the risk of the commodity, namely the parametric or nonparametric method. Thus, the methodology and the analysis of the results are based on measuring the market risk level for rice using the metric VaR and employing parametric and nonparametric calculation methods. According to the results, it was concluded that farmers are exposed to a high risk of price fluctuation, which can lead to financial losses and disinvestment in the sector. It was further concluded that the metric used is a valuable tool for the management of risk and that in the case of rice, only the nonparametric method is suitable for estimating the $\mathrm{VaR}$, since the price series did not show a normal distribution.
\end{abstract}

Key words: rice, price, value at risk.

\section{INTRODUÇÃO}

O gerenciamento de risco é valioso para os agricultores, visto que a gestão do risco permite a minimização das perdas financeiras e a melhor alocação dos recursos, reduzindo a probabilidade de prejuízos das empresas agrícolas.

No caso do agronegócio do arroz, essas análises são de grande importância, uma vez que além do preço do arroz possuir considerável volatilidade, o grão não possui um contrato futuro negociado na bolsa brasileira (CAPITANI et al., 2010), o que torna pertinente o uso e o desenvolvimento de mecanismos alternativos para o gerenciamento de risco da atividade orizícola. 
Assim, a importância do presente artigo está em identificar o nível de risco da commodity e com base nela alertar os agricultores e autoridades sobre as potencialidades de perdas e descapitalizações financeiras da atividade, bem como, justificar projetos que visem a implementação de mecanismos que minimizem os riscos do investimento no setor, como a implantação de contratos futuros para o grão na bolsa brasileira.

Nesse sentido, o artigo faz uma análise do risco da atividade orizícola aplicando a métrica Value at Risk (VaR). O estudo procura também avaliar a métrica como ferramenta para a gestão de risco pelos agricultores. O VaR é de fácil aplicação e pode contribuir no planejamento agrícola, favorecendo o agricultor na tomada das melhores decisões no gerenciamento do negócio.

Especificamente, o artigo teve dois objetivos, o primeiro foi avaliar o nível de risco que os produtores de arroz estão expostos no processo de comercialização da commodity. O segundo, por sua vez, consistiu em avaliar a aplicabilidade da métrica VaR na mensuração do risco do arroz e identificar qual a melhor maneira de se estimar o risco da commodity, pelo método paramétrico ou não paramétrico.

\section{Materiais e Métodos}

Para o melhor entendimento dos resultados, é apresentada nesse tópico a teoria sobre risco, sua definição, fonte, mensuração e forma de avaliação.

\subsection{Risco}

Risco é a possibilidade da ocorrência de um prejuízo financeiro, sendo muitas vezes definido como a variabilidade dos retornos dos ativos (GITMAN, 1997), sendo, para Woiler e Mathias (1996), devidos principalmente à grande quantidade de informação contida no projeto e de estimação dos valores utilizados para as variáveis, o que é inevitavelmente um risco, pois não se trabalha com valores reais. Segundo os autores, riscos podem ser classificados como internos e externos, sendo os primeiros provenientes de fontes endógenas e passíveis de influências empresariais, ao passo que os riscos externos são oriundos de fontes exógenas e as empresas possuem poucos meios para contorná-los.

Thiry-Cherques (2004) sugere as seguintes alternativas para minimização dos riscos: exaustiva análise das influências das externalidades no projeto, obtenção de informações históricas recentes no setor onde o projeto será concretizado e levantamento de dados de mercado e de informações comerciais.

Buarque (1991) apresenta três maneiras de reduzir os riscos:

a) Análise cuidadosa dos dados futuros, como as condições e possibilidades da propagação dos preços e insumos e da vida útil dos equipamentos.

b) Aplicação de dados conservadores quando o valor da variável for duvidoso.

c) Utilização de valores otimistas, realistas e pessimistas para as principais variáveis do projeto.

Com o propósito de minimizar os riscos, Woiler e Mathias (1996) sugerem: fazer estimativas mais cuidadosas; proceder a ajustes empíricos para não superestimar ou subestimar os valores; elaborar projeções pessimista, média e otimista das variáveis, promover o ajuste da taxa de desconto pelo risco, elevando a taxa para investimentos mais arriscados; fazer o ajuste pela incerteza equivalente; e promover análise de sensibilidade. Esses autores salientam ainda que a análise de risco é aperfeiçoada pelo emprego das simulações de Monte Carlo, baseadas na aleatoriedade dos valores das variáveis.

Diante do risco, os indivíduos podem apresentar três diferentes comportamentos: ser avesso, tendencioso ou indiferente (GITMAN, 1997). A Figura 1 demonstra de forma gráfica os diferentes comportamentos. 


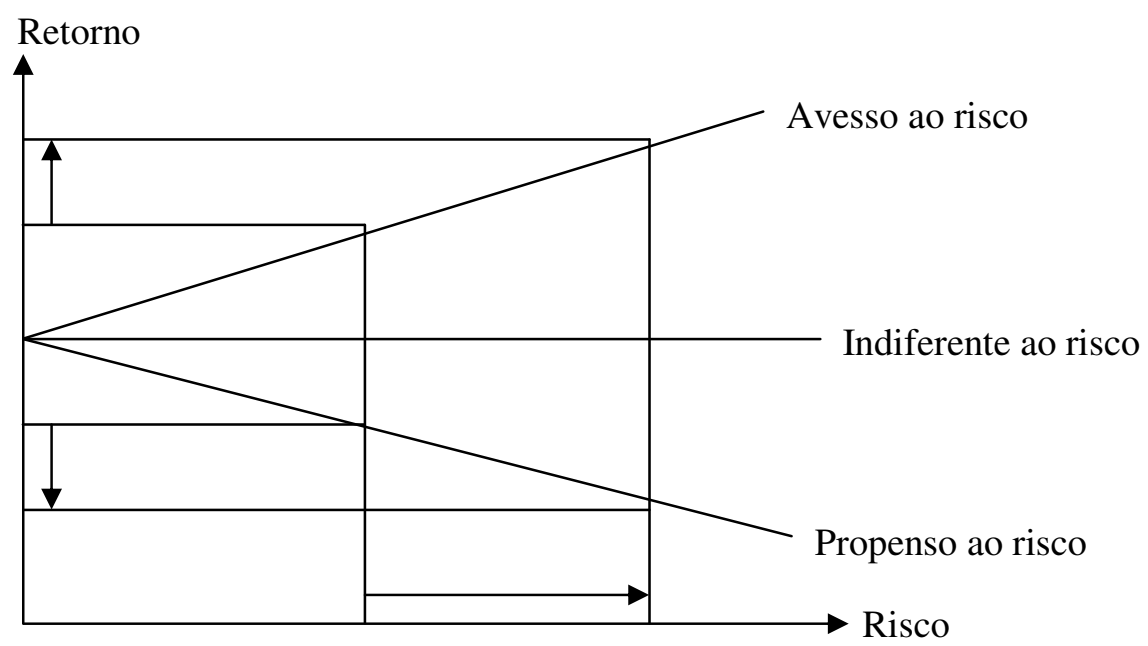

Fonte: Gitman (1997).

Figura 1 - Preferências e comportamentos em relação ao risco.

Pela Figura 1, quando o indivíduo é indiferente ao risco, nenhuma alteração no nível do retorno de um ativo é exigida quando ocorre elevação do risco. No entanto, alguns indivíduos são propensos ao risco, sendo atraídos pela sua elevação. Essa tendenciosidade leva o indivíduo a requerer maiores riscos, mesmo diante de menores retornos. Por outro lado, quando se é avesso ao risco, incrementos na taxa do retorno são exigidos na alta dos riscos; caso contrário, o ativo é desprezado, pois se exige uma compensação quando a possibilidade de perdas eleva-se. A maioria das pessoas se enquadra nesse tipo de comportamento conservador e não-agressivo (GITMAN, 1997). De modo geral, os indivíduos tendem a preferir ativos com maior relação retorno/risco aos ativos com menor retorno/risco.

Além disso, como as previsões futuras possuem maiores níveis de erros, com o passar do tempo, a variabilidade dos retornos dos ativos eleva-se com o tempo, caracterizando o risco como uma função crescente do tempo, como é visto na Figura 2.

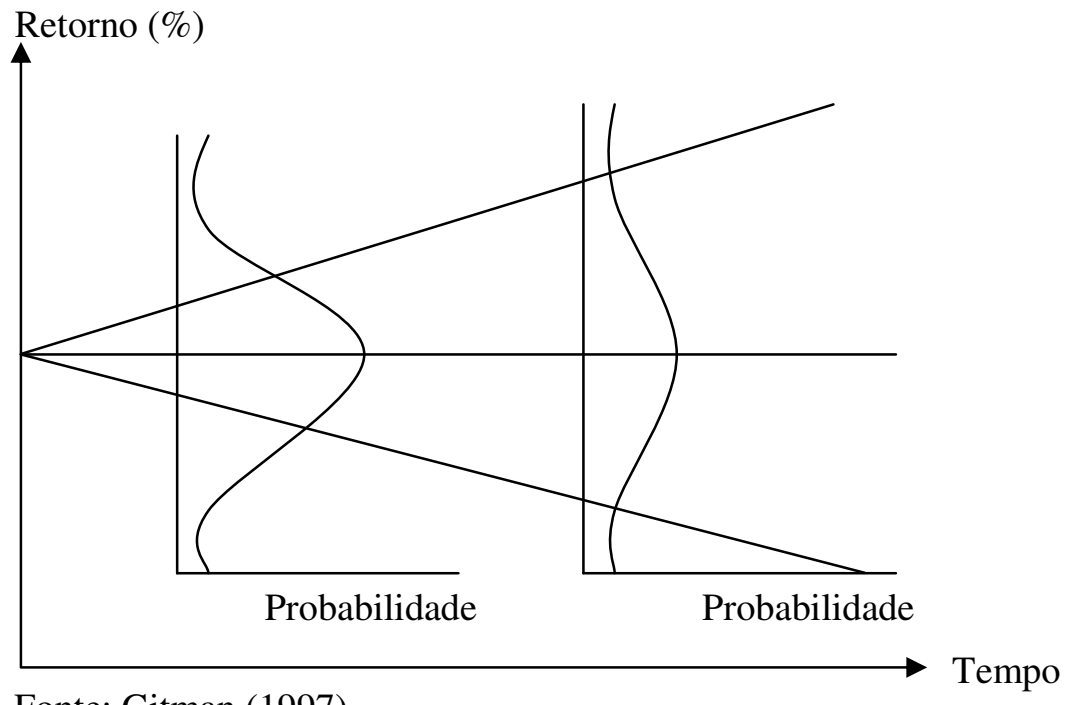

Fonte: Gitman (1997).

Figura 2 - Risco como função crescente do tempo. 
Os riscos podem ser mensurados quantitativamente pela transformação das possibilidades das perdas em probabilidades, sendo geralmente expressos por gráficos de barras, que relacionam cada taxa de retorno a cada probabilidade de ocorrência. Quando se tem todas as possíveis taxas de retorno e as respectivas probabilidades, o risco é melhor mensurado. Nesse caso, é viável expressar o risco pela distribuição de probabilidade contínua, construída a partir da distribuição de freqüência dos retornos passados, como é demonstrado na Figura 3 (GITMAN, 1997).

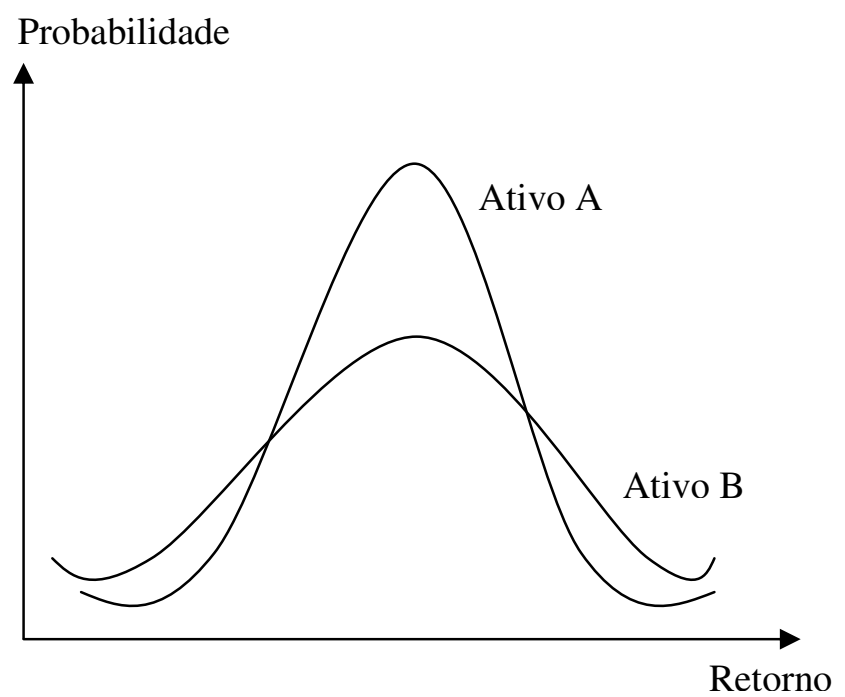

Fonte: Gitman (1997).

Figura 3 - Distribuição de probabilidade contínua do retorno do ativo.

Segundo Gitman (1997), o risco pode ser mensurado pelo desvio-padrão, medindo a dispersão dos retornos em relação ao seu valor esperado ou médio. Quanto maior o desvio, maior o risco do ativo. Na Figura 3, o ativo B apresenta maior risco que o ativo A, pois o retorno do ativo B possui maior variabilidade. O cálculo do desvio-padrão é dado pela equação:

$$
\sigma_{K}=\sqrt{\frac{\sum_{i=1}^{n}\left(K_{i}-\bar{K}\right)^{2}}{n-1}}
$$

em que $\sigma_{K}$ é o desvio-padrão dos retornos do ativo; K, os retornos para cada observação i; n, o número de observações analisadas; e $\bar{K}$, o retorno esperado, que é dado pela equação:

$$
\bar{K}=\sum_{i=1}^{n} K_{i} / n
$$

Ainda de acordo com Gitman (1997), quando se quer medir e comparar riscos de ativos com diferentes retornos, usa-se o indicador de coeficiente de variação, pois é mais indicado para análise da dispersão relativa dos retornos quando as médias dos retornos são diferentes. O coeficiente de variação é determinado pela equação:

$$
C V=\sigma_{k} / \bar{K}
$$


em que CV é o coeficiente de variação. Quanto maior o CV, maior o risco do ativo, visto que maior é a proporção do desvio-padrão em relação à média do retorno do ativo.

Conforme Ross et al. (1997), o risco pode ser sistemático e não-sistemático. O primeiro é o risco não diversificado ou de mercado, que influencia a grande maioria dos ativos. A formação de carteira de investimento e sua diversificação não eliminam esse tipo de risco. Por sua vez, a diversificação da carteira pela inclusão de novos ativos reduz o risco não-sistemático ou diversificado. Isso é possível porque, ao contrário do risco sistemático, o não-sistemático atinge apenas um pequeno grupo de ativos. Dessa forma, a diversificação das carteiras de investimento é eficaz na redução do risco, mas não totalmente, porque uma parte do risco é não-diversificada.

Uma representação dos efeitos da diversificação sobre as carteiras é mostrada na Figura 4, na qual se observa que o aumento do número de ativos na carteira promove a queda do risco, embora o risco sistemático não seja eliminado, pois ele influencia praticamente todos os ativos da carteira e não apenas um pequeno grupo desses (ROSS et al., 1997).

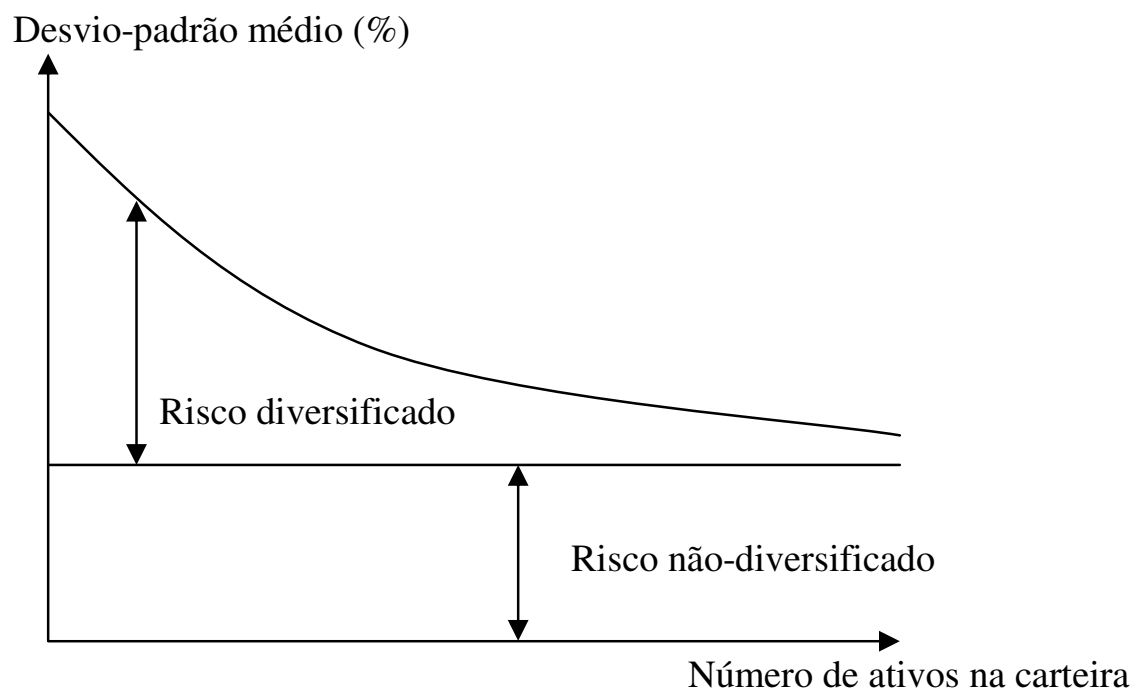

Fonte: Ross et al. (1997).

Figura 4 - Risco e diversificação de carteiras.

Tendo a diversificação a capacidade de eliminar o risco não-sistemático, o retorno esperado da carteira e de cada ativo depende exclusivamente do risco sistemático. Dessa forma, o ajuste do retorno da carteira ou do ativo em assumir risco deve ser efetuado apenas pelo risco sistemático (ROSS et al., 1997). Conforme esses autores, o retorno de um ativo i ajustado pelo nível de risco sistemático é dado pela equação:

$$
E\left(R_{i}\right)=R_{f}+\beta_{i}\left[E\left(R_{m}\right)-R_{f}\right]
$$

em que $E\left(R_{i}\right)$ é o retorno esperado de um ativo i; $R_{f}$, o retorno livre de risco; $\beta_{i}$, o nível de risco sistemático do ativo; $E\left(R_{m}\right)$, o retorno esperado do mercado; e $E\left(R_{m}\right)-R_{f}$, o prêmio por assumir risco.

Segundo Varian (2003), a condição para equilíbrio no mercado de ativo é que o retorno esperado dos ativos, que são ajustados pelo risco, seja igual, pois ativos que possuem maior relação retorno/risco 
despertam um rápido interesse dos indivíduos, fazendo com que seu preço suba e apresente a mesma relação retorno/risco que os demais ativos do mercado.

Conforme Varian (1993), a equação conhecida como CAPM (Capital Asset Precing Model), derivase do ponto de interseção da reta orçamentária e da curva de indiferença do comportamento do indivíduo em relação ao risco. Nesse ponto, a Taxa Marginal de Substituição (TMS) entre risco e retorno é igual à inclinação da reta orçamentária, sendo essa inclinação o preço do risco.

A Figura 5 demonstra o ponto de interseção das curvas para um indivíduo avesso ao risco. A curva de indiferença mede as preferências do indivíduo em relação ao retorno e ao risco, enquanto a reta orçamentária expressa o custo em conseguir maior retorno dado à elevação do risco, medida pelo desviopadrão do retorno (VARIAN, 1993).

Na Figura 5 é demonstrado o ponto de interseção das curvas para um indivíduo avesso ao risco. A curva de indiferença mede as preferências do indivíduo em relação ao retorno e ao risco, enquanto a reta orçamentária expressa o custo em conseguir maior retorno dado à elevação do risco, medida pelo desviopadrão do retorno (VARIAN, 1993).

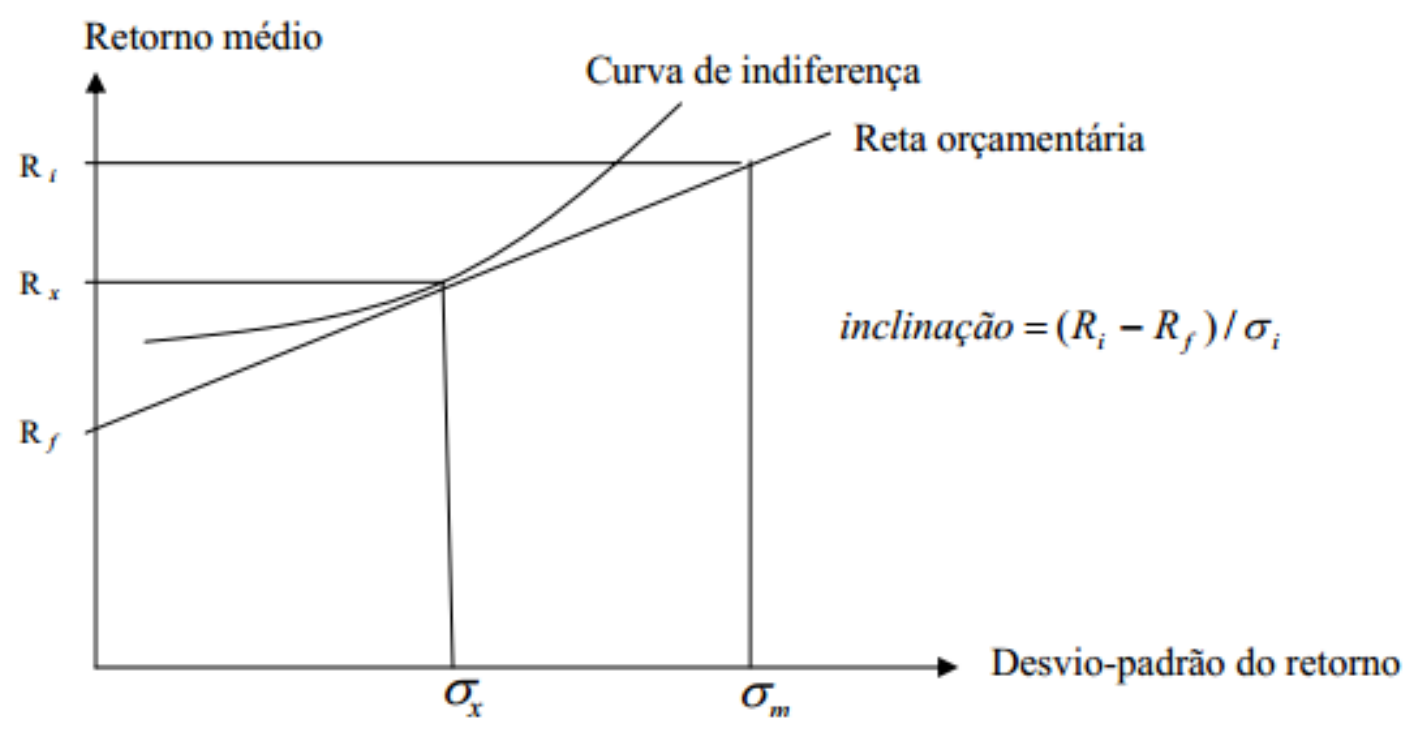

Fonte: Varian (1993).

Figura 5 - Risco e retorno.

Matematicamente, no ponto de escolha ótima entre retorno e risco tem-se:

$$
p=T M S=\frac{\left(R_{i}-R_{f}\right)}{\sigma_{i}}
$$

em que p é o preço do risco; TMS, a taxa marginal de substituição entre risco e retorno; $\mathrm{R}_{\mathrm{i}}$, o retorno do ativo de risco; $\mathrm{R}_{\mathrm{f}}$, o retorno livre de risco; e $\sigma_{i}$, o desvio-padrão dos retornos do mercado. 


\subsection{Referencial analítico}

Como método de análise, o artigo utiliza a métrica Value at Risk (VAR) e indicadores estatísticos, como: desvio-padrão, assimetria e curtose. É realizado também o teste de normalidade de Jarque-Bera (JB). Dada a importância desses indicadores na mensuração do risco e o seu uso no artigo, segue abaixo a apresentação e discussão dos indicadores.

\subsubsection{O Value at Risk (VAR)}

O Valor em Risco (VaR - Value at Risk) é uma ferramenta de gerenciamento do risco que tem se difundido cada vez mais no mercado financeiro. A métrica informa o risco de uma carteira, empresa ou instituição financeira em um único número (PAMPLONA, 2003).

O VaR foi desenvolvida pelos executivos do Banco J. P. Morgan, na década de 1980, cuja intenção era medir o risco potencial de uma perda através de um único indicador em um dado intervalo de tempo a um nível determinado de significância (JORION, 2003), na forma de equação:

$$
\operatorname{Pr}\left(\Delta R_{t} \leq \operatorname{VaR}_{t}\right)=\alpha \%
$$

em que $\operatorname{Pr}$ é a probabilidade de ocorrência de uma perda máxima dada pela $\Delta R_{t}$, variação monetária do ativo ou portfólio, em um instante $t$ ao nível de significância $\alpha$.

Em seu estudo Rugani e Silveira (2006) estimaram a máxima perda potencial e calcularam a relação entre o VaR e o valor investido. Conforme esses autores, essa relação permite obter o Percentual de Risco, isto é, o risco absorvido pelo agente.

Em termos de equação, a relação entre o VaR e o valor investido é escrita como:

$$
P R=\frac{V a R}{I} x 100
$$

em que PR é o Percentual de Risco ou VaR em percentagem do valor investido; VaR é o Value at Risk; e I, o nível de investimento realizado pelo agente. Quanto maior o PR, maior a exposição ao risco.

Para o cálculo do VaR, existem duas formas: a maneira paramétrica e a não paramétrica. Na primeira, é pressuposto determinada distribuição de probabilidade (normal ou log normal) e usadas medidas estatísticas como variância, desvio-padrão e covariância para mensuração dos riscos dos ativos e dos portfólios. Já para o método não paramétrica ou de simulação, não se faz a priori pressuposições sobre o comportamento das variáveis, isto é, não se pressupõe a distribuição de probabilidade nem correlação (SILVA NETO, 2002).

Conforme Silva Neto (2002), o VaR pode ser calculado usando o método paramétrico normal e os métodos não paramétricos de Simulação Histórica e de Monte Carlo. Porém, como muitos ativos não apresentam uma distribuição normalmente distribuída, tem-se buscado superar esse problema aplicando-se os modelos não paramétricos.

Segundo Silva Neto (2002), cada modelo (paramétrico e não paramétrico) possui vantagens e desvantagens, sendo a decisão de uso desses modelos uma opção individual. No entanto, os modelos não paramétricos, embora mais complicados de implementação e de necessitarem de mais processamento, tem 
ganhado espaço, sendo a principal vantagem desses modelos o fato de adaptarem aos retornos de ativos que afastam da distribuição normal. ${ }^{1}$

\subsubsection{Indicadores estatísticos}

A primeira etapa do trabalho foi verificar as propriedades estatísticas da série de preços do arroz. Para isso, obteve-se informações sobre a sua distribuição histórica no período em estudo, através de seus valores médio, máximo e mínimo; e de seus valores estatísticos: desvio-padrão, assimetria, curtose. Além disso, promoveu-se o teste estatístico de normalidade de Jarque-Bera (JB) para verificar se a série de preços é normalmente distribuída.

Para o cálculo do preço médio e desvio-padrão da série de preços do arroz, foram aplicadas as equações:

$$
\begin{gathered}
\bar{P}=\sum_{i=1}^{n} P_{i} / n \\
\sigma_{P}=\sqrt{\frac{\sum_{i=1}^{n}\left(P_{i}-\bar{P}\right)^{2}}{n-1}}
\end{gathered}
$$

em que P é o valor do preço para cada observação $i$; $n$, o número de observações; $\bar{P}$, o valor médio dos preços; e $\sigma_{p}$, o desvio-padrão da série de preços.

Para completar a análise, foi obtido também os valores da simetria e da curtose e realizado o teste de JB para a distribuição da série de preços. Assim, verificou se a série obedece a função de distribuição normal:

$$
f(x, \mu, \sigma)=\frac{1}{\sqrt{2 \pi \sigma^{2}}} e^{-\left[\frac{(x-\mu)^{2}}{2 \sigma^{2}}\right]}
$$

em que $\mu$ e $\sigma$ são os dois primeiros momentos da distribuição, sendo o primeiro a média e o segundo o desvio-padrão e $e^{y}$ a exponencial (GUJARATI, 2000).

Para testar a hipótese de normalidade da distribuição, foi empregado o teste estatístico de JB:

$$
J B=n\left[\frac{S^{2}}{6}+\frac{(C-3)^{2}}{24}\right]
$$

em que é testado a hipótese nula de normalidade, tendo JB distribuição qui-quadrado com 2 graus de liberdade. Na equação, $\mathrm{S}$ é a Assimetria e $\mathrm{C}$ a Curtose da distribuição. Especialmente no caso da distribuição normal, quando $S=0$ e $C=3$, se diz que a distribuição é normal, simétrica e mesocúrtica (GUJARATI, 2000).

Caso a série de preços tiver distribuição normal, a sua assimetria deverá ser próxima de zero e a curtose próxima de três, podendo a função de distribuição normal ser empregada na estimação do VaR pelo

\footnotetext{
${ }^{1}$ Para um melhor entendimento dos modelos paramétricos e não paramétricos para o cálculo do Value at Risk (VaR) ver Silva Neto (2002), pag. 232-248.
} 
método paramétrico. Caso a série de preços não tiver distribuição normal, irá ser aplicado apenas os métodos não paramétricos para cálculo do VaR.

\subsubsection{Procedimentos e fonte de dados}

Para a realização das estimativas foi utilizada a série de preços mensal do quilo do arroz em casca ao produtor no Estado de São Paulo no período de janeiro de 1995 a fevereiro de 2012. A série de preços foi coletada junto ao Instituto de Economia Agrícola (IEA).

\section{RESULTADOS E DISCUSSÃO}

Antes de estimar o VaR, foram obtidos os dados estatísticos da série de preços do quilo do arroz ao nível do produtor, no período de janeiro de 1995 a fevereiro de 2012. Analisando as propriedades estatísticas da série de preços do arroz, notou-se que o preço médio do $\mathrm{Kg}$ recebido pelo produtor foi de $\mathrm{R} \$ 0,41$, tendo o preço ao produtor alcançado o preço mínimo de $\mathrm{R} \$ 0,15$ e o máximo de $\mathrm{R} \$ 0,71$. Em relação a dispersão dos preços, dada pelo desvio-padrão, a série de preços oscilou cerca de $\mathrm{R} \$ 0,16$ (Tabela 1).

Tabela 1: Análise das propriedades estatísticas das séries de preços do arroz, 01/1995 a 2/2012.

\begin{tabular}{ll}
\hline Indicador & Valor \\
\hline Média & 0,4141 \\
Máximo & 0,7100 \\
Mínimo & 0,1500 \\
Desvio-padrão & 0,1587 \\
Assimetria & 0,1005 \\
Curtose & 1,6967 \\
Jarque-Bera (JB) & 14,925 \\
Probabilidade $(\mathrm{JB})$ & 0,0006
\end{tabular}

Fonte: Resultados da pesquisa obtidos usando a série de preços.

Além disso, pela Tabela 1, pode-se verificar pelo teste de Jarque-Bera (JB) que a série de preços não é normalmente distribuída, sendo rejeitada a hipótese nula de normalidade, uma vez que a estatística calculada foi maior que o valor tabelado da estatística qui-quadrado com 2 graus de liberdade e $10 \%(4,61)$.

Conforme Gujarati (2000), se o valor p (probabilidade) da estatística qui-quadrado calculada for suficientemente baixa, pode-se rejeitar a hipótese de normalmente. No caso da atual série de preços, o valor $\mathrm{p}$ da estatística foi de apenas 0,0006. Adicionalmente, o valor da Assimetria e da Curtose foram de 0,1005 e 1,6967, diferentes dos valores condizentes de uma série com distribuição normal.

Nesse sentido, dado que a série não se distribui normalmente, o cálculo do VaR foi realizado usando apenas os métodos não paramétricos de Simulação Histórica e de Monte Carlo. Na Tabela 2 estão os valores do VaR calculados pelo método de Simulação Histórica para a posição de comercialização de 100, 500 e 1000 sacas de arroz de $60 \mathrm{~kg}$, aos níveis de confiança de 99\%, 95\% e 90\%. 
Os resultados na Tabela 2 mostram que para o agricultor que possui 100 sacas de arroz ele tem a preços de fevereiro de 2012 um valor investido de $\mathrm{R} \$ 3.223,00$, estando sujeito a uma perda máximo de $\mathrm{R} \$$ 897,00 em um mês, com $99 \%$ de confiança. Porém, quando o nível de confiança passa para 95\% e 90\%, os riscos de perdas financeiras aumentam para $\mathrm{R} \$ 1.138,00$ e $\mathrm{R} \$ 1.239,00$. Interpretação simular é feita para o investimento em 500 sacas e para o investimento em 1.000 sacas.

Tabela 2: Value at Risk (VaR) obtido pelo método de Simulação Histórica.

\begin{tabular}{lccc}
\hline \multirow{2}{*}{ Nível de significância } & \multicolumn{3}{c}{ Posição } \\
\cline { 2 - 4 } & $100 \mathrm{sc}$ & $500 \mathrm{sc}$ & $1000 \mathrm{sc}$ \\
\hline $99 \%$ & $\mathrm{R} \$ 897,00$ & $\mathrm{R} \$ 4.485,00$ & $\mathrm{R} \$ 8.970,00$ \\
$95 \%$ & $\mathrm{R} \$ 1.138,00$ & $\mathrm{R} \$ 5.690,00$ & $\mathrm{R} \$ 11.380,00$ \\
$90 \%$ & $\mathrm{R} \$ 1.239,00$ & $\mathrm{R} \$ 6.195,00$ & $\mathrm{R} \$ 12.390,00$ \\
\hline Valor de mercado & $\mathrm{R} \$ 3.223,00$ & $\mathrm{R} \$ 16.115,00$ & $\mathrm{R} \$ 32.230,00$ \\
\hline
\end{tabular}

Fonte: Resultados obtidos pelo processamento da série de preços.

Já na Tabela 3 estão os valores do VaR calculados pelo método de Monte Carlo. Comparando esse método com o de Simulação Histórica, nota-se que o risco mensurado pelo método de Monte Carlo fornece valores ligeiramente maiores. Por exemplo, no caso do investimento em 1.000 sacas, o risco identificado pelo método de Monte Carlo foi de R \$12.471,13 ao nível de confiança de $90 \%$, contra R\$12.390,00 identificados pelo método de Simulação Histórica.

Tabela 3: Value at Risk (VaR) obtido pelo método de Monte Carlo.

\begin{tabular}{lccc}
\hline \multirow{2}{*}{ Nível de significância } & \multicolumn{3}{c}{ Posição } \\
\cline { 2 - 4 } & $100 \mathrm{sc}$ & $500 \mathrm{sc}$ & $1000 \mathrm{sc}$ \\
\hline $99 \%$ & $\mathrm{R} \$ 956,57$ & $\mathrm{R} \$ 4.782,85$ & $\mathrm{R} \$ 9.565,70$ \\
$95 \%$ & $\mathrm{R} \$ 1.115,56$ & $\mathrm{R} \$ 5.577,80$ & $\mathrm{R} \$ 11.155,60$ \\
$90 \%$ & $\mathrm{R} \$ 1.247,11$ & $\mathrm{R} \$ 6.235,57$ & $\mathrm{R} \$ 12.471,13$ \\
\hline Valor de mercado & $\mathrm{R} \$ 3.223,00$ & $\mathrm{R} \$ 16.115,00$ & $\mathrm{R} \$ 32.230,00$
\end{tabular}

Fonte: Resultados obtidos pelo processamento da série de preços.

Uma melhor visualização do grau de risco identificado pelos métodos é visto nas Tabelas 4 e 5 . Pelas Tabelas, os agricultores estão consideravelmente expostos aos riscos de perdas financeiras e, consequentemente, o setor está sujeito a descapitalização, comprometendo a produção e o consumo de um importante alimento pelas famílias brasileiras, prejudicando especialmente as classes sociais mais carentes.

Segundo o método de Simulação Histórica (Tabela 4), o agricultor que possui um investimento em 100 sacas está sujeito a uma perda de investimento de $28 \%$ ao nível de confiança de $99 \%$. Porém, para agricultores menos avessos ao risco, o percentual de risco é de 35\% e 38\%, ao nível de segurança de $95 \%$ e $90 \%$. 
Tabela 4: Value at risk (VaR) em percentual obtido pelo método de Simulação Histórica.

\begin{tabular}{lccc}
\hline \multirow{2}{*}{ Nível de significância } & \multicolumn{3}{c}{ Posição } \\
\cline { 2 - 4 } & $100 \mathrm{sc}$ & $500 \mathrm{sc}$ & $1000 \mathrm{sc}$ \\
\hline $99 \%$ & $28 \%$ & $28 \%$ & $28 \%$ \\
$95 \%$ & $35 \%$ & $35 \%$ & $35 \%$ \\
$90 \%$ & $38 \%$ & $38 \%$ & $38 \%$
\end{tabular}

Fonte: Resultados obtidos pelo processamento da série de preços.

De forma semelhante, o método de Monte Carlo apresenta um percentual de risco de 30\%, 35\% e $39 \%$, aos níveis de confiança de $99 \%, 95 \%$ e $90 \%$ (Tabela 5). Assim, identifica-se que os métodos foram semelhantes na estimação do grau de risco, embora o método de Monte Carlo tenha capturado um nível de risco um pouco maior.

Tabela 5: Value at risk (VaR) em percentual obtido pelo método de Monte Carlo.

\begin{tabular}{lccc}
\hline $\begin{array}{l}\text { Nível de } \\
\text { significância }\end{array}$ & & Posição & \\
\cline { 2 - 4 } & $100 \mathrm{sc}$ & $500 \mathrm{sc}$ & $1000 \mathrm{sc}$ \\
\hline $99 \%$ & $30 \%$ & $30 \%$ & $30 \%$ \\
$95 \%$ & $35 \%$ & $35 \%$ & $35 \%$ \\
$90 \%$ & $39 \%$ & $39 \%$ & $39 \%$ \\
\hline
\end{tabular}

Fonte: Resultados obtidos pelo processamento da série de preços.

No caso do café, Rugani e Silveira (2006) estimaram, para o período de 1980 a 2005, pelo método de Simulação Histórica, um VaR de 24,60\% ao nível de confiança de $99 \%$ para os cafeicultores na região do Sul de Minas Gerais. Assim, segundo os autores, para o investimento de US\$25.000,00 em sacas de café, existe $1 \%$ de chance das perdas serem no máximo igual a US\$ 6.151,05.

Por fim, realizou-se uma análise do risco em três cenários na comercialização de arroz. No primeiro cenário, considerou-se que o agricultor tenha $\mathrm{R} \$ 10.000,00$ investidos em sacas de arroz; no segundo cenário, considerou-se que foram investidos $\mathrm{R} \$ 25.000,00$; e no terceiro, considerou-se que foram investidos $\mathrm{R} \$ 50.000,00$. Pela Figura 6, observa-se que ao nível de segurança de $99 \%$ os dois métodos forneceram estimativas próximas de risco. Por exemplo, quando foram investidos $\mathrm{R} \$ 10.000,00$, o método de Simulação Histórica e de Monte Carlo, estimaram perdas máximas de aproximadamente R \$ 2.500,00. 


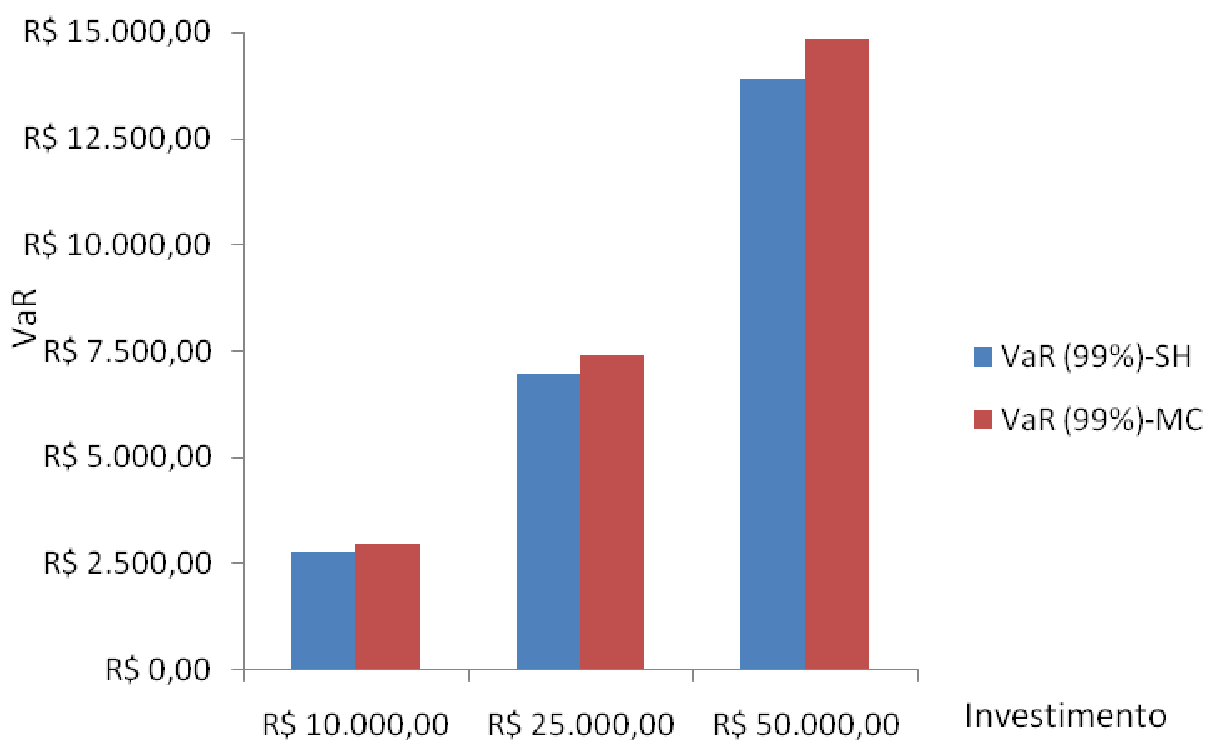

Fonte: Resultados obtidos pelo processamento da série de preços. (MC)

Figura 6 - Value at risk (VaR) obtido pelos métodos de Simulação Histórica (SH) e de Monte Carlo

Comparando esses resultados com os encontrados por Rugani e Silveira (2006) para o investimento em sacas de café, constata-se que o investimento em sacas de arroz é de alto risco, quando comparado ao risco do preço do café.

Conforme Capitani et al. (2010), embora o arroz seja uma das principais commodities produzida e consumida no Brasil e possua importância na segurança alimentar, ainda não possui um contrato futuro negociando na bolsa do país.

Além disso, os autores notaram que no período de setembro de 2005 a dezembro de 2009 o preço do arroz teve a maior volatilidade $(26,23 \%)$ quando comparado com as principais commodities agrícolas negociadas na BM\&F, a saber: café, milho, soja, açúcar e boi-gordo, respectivamente. O que evidencia a necessidade de mecanismos de proteção de risco por parte dos produtores e indústria orizícola no país, como um contrato futuro (CAPITANI et al., 2010).

\section{CONCLUSÃO}

Para continuar no mercado, cada vez mais é exigido o aperfeiçoamento das técnicas de produção e de gerenciamento da atividade, entre elas a adoção de sistemas de gerenciamento de risco que permitem a minimização do risco da atividade, como é o caso dos contratos futuros, e de ferramentas que possibilitem a previsão de perdas, como é o caso da métrica Value at Risk (VaR).

Nesse sentido, dada a importância do monitoramento dos preços agrícolas, o trabalho avaliou o nível de risco que os produtores de arroz estão expostos no processo de comercialização da commodity utilizando a métrica VaR.

Conforme os resultados, os agricultores estão expostos a um alto risco de oscilação de preços, o que pode levar a perdas financeiras e, consequentemente, a descapitalização do setor, o comprometimento da 
renda agrícola e a segurança alimentar, pois afeta principalmente as famílias mais carentes, que tem no arroz um dos principais alimentos.

Foi verificado também, que a métrica VaR pode ser aplicada ao gerenciamento do risco da commodity, permitindo o melhor planejamento das atividades e a minimização dos riscos. Porém, para o caso do arroz, apenas o método não paramétrico é indicado para a estimação da métrica, uma vez que a série de preços não apresentou uma distribuição normal.

\section{REFERÊNCIAS}

BUARQUE, C. Avaliação econômica de projetos: uma apresentação didática. $8^{a}$ ed. Rio de Janeiro: Campus, 1991. $266 \mathrm{p}$.

CAPITANI, D. H. D.; REGAZZINI, L. C.; MATTOS, F.L. Viabilidade de implantação de um contrato futuro de arroz no Brasil: uma análise dos pré-requisitos necessários. In: XLVIII Congresso da Sociedade Brasileira de Economia, Administração e Sociologia Rural, 2010, Campo Grande - MS. Anais do XLVIII Congresso da Sociedade Brasileira de Economia, Administração e Sociologia Rural, 2010.

GITMAN, L. J. Princípios de administração financeira. $7^{\text {a }}$ ed. São Paulo: Harbra, 1997.

GUJARATI, D. N. Econometria básica. $3^{\mathrm{a}}$ ed. São Paulo: Pearson Makron Books, 2000. 846 p.

JORION, P. Value at Risk: a nova fonte de refrência para a gestão do risco financeiro. São Paulo: Bolsa de Mercadorias e Futuros. $2^{\mathrm{a}}$ ed., 2003.

PAMPLONA, E. O. Gerenciamentos de Riscos em Custos. VIII Congresso Internacional de Costos. Punta Del Leste, Uruguay, 26 a 28 de novembro de 2003.

ROSS, S. A.; WESTERFIELD, R. W.; JORDAN, B. D. Princípios de administração financeira. $2^{\mathrm{a}}$ ed. São Paulo: Atlas, 1997. 425 p.

RUGANI, F. L.; SILVEIRA, S. F. R. Análise de risco para o café em Minas Gerais. Revista de Economia e Agronegócio, v. 4, p. 343-364, 2006.

SILVA NETO, L. A. Derivativos: definições, emprego e risco. $4^{a}$ ed., São Paulo: Atlas, 2002, 298 p.

THIRY-CHERQUES, H. R. Modelagem de projetos. 2a ed. São Paulo: Atlas, 2004. 265 p.

VARIAN, H. R. Intermediate microeconomics: a modern approach. New York: W. W. Norton e Company. 1993. $3^{\circ}$ ed., 623p.

WOILER, S.; MATHIAS, W. F. Projetos: planejamento, elaboração e análise. São Paulo: Atlas, 1996. 294 p. 